\title{
Razumikhin-type Theorems for ISS of Nonlinear Delayed Impulsive Systems
}

\author{
Wu-Hua Chen \\ College of Mathematics and Information Science \\ Guangxi University \\ Nanning, Guangxi, 530004 \\ P. R. China
}

\author{
Wei Xing Zheng \\ School of Computing and Mathematics \\ University of Western Sydney \\ Penrith South DC NSW 1797 \\ Australia
}

\begin{abstract}
In this paper we investigate the problem of inputto-state stability (ISS) of nonlinear delayed impulsive systems. Both the continuous dynamics and the discrete dynamics of nonlinear impulsive systems are subjected to external input. By virtue of the Razumikhin technique in combination with Lyapunov functions, we obtain some Razumikhin-type theorems that warrant input-to-state stability of nonlinear impulsive systems with time-delays. The Razumikhin-type input-to-state stability theorems cover the cases where the delayed continuous dynamics are input-to-state stable or destabilizing and the discrete dynamics are input-to-state stable or destabilizing, such that nonlinear delayed impulsive systems are able to retain input-to-state stability under certain conditions. The applicability of the derived Razumikhin-type theorems is illustrated by numerical results.
\end{abstract}

\section{INTRODUCTION}

In 1989 in his seminal paper [1], Sontag first introduced the concept of input-to-state stability (ISS). It is now widely accepted that the input-to-state stability property provides a natural framework for formulating notions of stability with respect to input perturbations, that is, for characterizing the continuity of state trajectories on the initial states and the external inputs. Roughly speaking, the input-to-state stability property means that no matter what the initial state is, if the external input is small, then the state must be eventually small. Input-to-state stability has been proven useful in the analysis and design of nonlinear control systems (see, e.g., [2]-[6]). Input-to-state stability was originally defined for continuous-time systems. Various extensions of the inputto-state stability property have since been made for different types of dynamical systems, for instance, discrete-time systems [7], switched systems [8], time-delay systems [9]-[10], stochastic systems [11], etc.

In practical situations, many processes are subjected to short temporary perturbations, called impulses, which are negligible compared to the process duration. Impulsive systems provide a natural description of observed processes with impulse effect. For example, networked control systems with the Return Routability (RR) or try-once-discard (TOD) dynamic protocol can be modeled by impulsive systems [12][13]; in the analysis of switched systems using multiple Lyapunov functions, the evolution of multiple Lyapunov functions along the state trajectories can be also described by impulsive systems; and when applying an impulsive control law to stabilize unstable continuous systems, the resulting control system is an impulsive system. An impulsive system can be viewed as a hybrid one that consists of continuous dynamics, discrete dynamics, and a criteria for determining when the states of the system are to be reset. The stability of impulsive systems has been extensively studied in the literature (we refer to [16]-[17] and the references therein). $H_{\infty}$ control problems for three types of linear delay-free impulsive systems were studied in [19]. Recently, the concept of input-to-state stability for nonlinear delay-free impulsive systems has been introduced in [20], and a set of Lyapunovbased sufficient conditions for these properties have been 
established. The results in [20] have shown that when one of the continuous dynamics and the discrete dynamics that govern the impulses are input-to-state stable but the other are not, the impulsive system can maintain input-to-state stability under some constraint conditions on impulsive interval.

In real systems such as engineering, biological, and economical systems, very often there exist input delays. However, due to the presence of an input delay, the performance of the system is frequently adversely affected. In [9] and [10], Razumikhin technique and Lyapunov functional method were applied to study the input-to-state stability property for nonlinear time-delay systems. However, it seems that there have been no available results that consider the effect of both impulses and time-delay on input-to-state stability for nonlinear systems, which still remains an important and open problem.

This paper is devoted to the investigation of the inputto-state stability property for nonlinear delayed impulsive systems with external input affecting both the continuous dynamics and the discrete dynamics. The primary objective is to derive Razumikhin-type theorems for guaranteeing the input-to-state stability property. It is shown that when the delayed continuous dynamics are input-to-state stable and the discrete dynamics are destabilizing, the input-to-state stability property of the original impulsive systems can be maintained if the length of impulsive interval is large enough. Conversely, when the discrete dynamics are input-to-state stable but the delayed continuous dynamics are not, the impulsive system can attain input-to-state stability if the sum of the length of impulsive interval and the time-delay is small enough.

\section{SYSTEM DESCRIPTION}

In the sequel, if not explicitly stated, matrices are assumed to have compatible dimensions. The notation $M>$ $(\geq,<, \leq) 0$ is used to denote a symmetric positive-definite (positive-semidefinite, negative, negative-semidefinite) matrix. $\lambda_{\min }(\cdot)$ and $\lambda_{\max }(\cdot)$ represent the minimum and maxi- mum eigenvalues of the corresponding matrix, respectively. $|\cdot|$ denotes the Euclidean norm for vectors or the spectral norm for matrices. For $\tau>0$, let $P C\left([-\tau, 0], \mathbb{R}^{n}\right)$ denote the set of piecewise right continuous function

$$
\phi:[-\tau, 0] \rightarrow \mathbb{R}^{n}
$$

with the norm defined by

$$
\|\phi\|_{\tau}=\sup _{-\tau \leq \theta \leq 0}\|\phi(\theta)\|
$$

If

$$
x \in P C\left(\left[t_{0}-\tau,+\infty\right), \mathbb{R}^{n}\right),
$$

then for each $t \geq t_{0}$, we define

$$
x_{t} \in P C\left([-\tau, 0], \mathbb{R}^{n}\right)
$$

by

$$
x_{t}(s)=x(t+s)
$$

for $-\tau \leq s \leq 0$. Let $\mathbb{N}$ denote the set of positive integers, i.e.,

$$
\mathbb{N}=\{1,2, \ldots\}
$$

Consider the nonlinear delayed impulsive systems of the form:

$$
\begin{aligned}
\dot{x}(t) & =f\left(x_{t}, w_{c}(t)\right), \quad t>t_{0}, t \neq t_{k}, \\
x(t) & =g\left(x\left(t^{-}\right), w_{d}\left(t^{-}\right)\right), \quad t=t_{k}, \\
x_{t_{0}} & =\phi
\end{aligned}
$$

where $x(t) \in \mathbb{R}^{n}$ is the system state, $w_{c}(t) \in \mathbb{R}^{m_{1}}$ is a locally bounded external input, $w_{d}(t) \in \mathbb{R}^{m_{2}}$ is the impulsive disturbance input, and $\phi \in P C\left([-\tau, 0], \mathbb{R}^{n}\right)$ is the initial condition of the state.

$$
f: P C\left([-\tau, 0] \times R^{m_{1}} \rightarrow \mathbb{R}^{n}\right.
$$

and

$$
g: \mathbb{R}^{n} \times \mathbb{R}^{m_{2}} \rightarrow \mathbb{R}^{n}
$$

satisfy

$$
f(0,0)=g(0,0)=0 .
$$


$\left\{t_{k}\right\}$ is a strictly increasing sequence of impulse times in $\left(t_{0},+\infty\right)$ and satisfies

$$
\lim _{k \rightarrow+\infty} t_{k}=+\infty .
$$

We assume the following hypotheses throughout our paper:

$\left(\mathbf{A}_{1}\right) \tilde{f}(t, \psi)=f\left(\psi, w_{c}(t)\right)$ is composite-PC, i.e., for each $t_{0} \in \mathbb{R}^{+}$and $\sigma>0$, if

$$
x \in P C\left(\left[t_{0}-\tau, t_{0}+\sigma\right], \mathbb{R}^{n}\right)
$$

and $x$ is continuous at each $t \neq t_{k}$ in $\left(t_{0}, t_{0}+\sigma\right]$, then the composite function $h$ defined by

$$
h(t)=\tilde{f}\left(t, x_{t}\right)
$$

is an element of the function class $P C\left(\left[t_{0}, t_{0}+\sigma\right], \mathbb{R}^{n}\right)$.

(A $\left.\mathbf{A}_{2}\right) \tilde{f}(t, \psi)$ is quasi-bounded, i.e., for each $t_{0} \in \mathbb{R}^{+}$and $\sigma>0$, and for each compact set $F \in \mathbb{R}^{n}$, there exists some $M>0$ such that

$$
|\tilde{f}(t, \psi)| \leq M
$$

for all

$$
(t, \psi) \in\left[t_{0}, t_{0}+\sigma\right] \times P C([-\tau, 0], F) .
$$

(A $\left.\mathbf{A}_{3}\right)$ For each fixed $t \in \mathbb{R}^{+}, \tilde{f}(t, \psi)$ is a continuous function of $\psi$ on $P C\left([-\tau, 0], \mathbb{R}^{n}\right)$.

It is shown in [18] that under Assumptions $\left(\mathbf{A}_{1}\right)-\left(\mathbf{A}_{3}\right)$, the initial value problem (1) admits a solution $x\left(t, t_{0}, \phi\right)$ that exists in a maximal interval $I$. In addition, if $\tilde{f}(t, \psi)$ is locally Lipschitz in $\psi$, then the solution is unique.

We define the following function classes. A function $\alpha: \mathbb{R}^{+} \rightarrow \mathbb{R}^{+}$is of class $\mathcal{K}$, if $\alpha$ is continuous, strictly increasing, and $\alpha(0)=0$. If $\alpha$ is also unbounded, then we say that it is of class $\mathcal{K}_{\infty}$. A function

$$
\varpi: \mathbb{R}^{+} \times \mathbb{R}^{+} \rightarrow \mathbb{R}^{+}
$$

is of class $\mathcal{K} \mathcal{L}$, if $\varpi(\cdot, t)$ is of class $\mathcal{K}$ in the first argument for each fixed $t \geq 0$ and $\varpi(s, t)$ decreases to 0 as $t \rightarrow+\infty$ for each fixed $s \geq 0$.

Definition 1: Suppose that a sequence $\left\{t_{k}\right\}$ is given. System (1) is said to be input-to-state stable (ISS) if there exist functions $\varpi \in \mathcal{K} \mathcal{L}$ and $\gamma_{c}, \gamma_{d} \in \mathcal{K}_{\infty}$ such that for every initial condition and every pair of input $\left(w_{c}, w_{d}\right)$, the solution of system (1) exists globally and satisfies

$$
\begin{aligned}
|x(t)| \leq & \varpi\left(\|\phi\|_{\tau}, t-t_{0}\right)+\gamma_{c}\left(\left\|w_{c}\right\|_{\left[t_{0}, t\right]}\right) \\
& +\gamma_{d}\left(\max _{t_{k} \in\left[t_{0}, t\right]}\left\{\left|w_{d}\left(t_{k}^{-}\right)\right|\right\}\right), \quad t \geq t_{0},
\end{aligned}
$$

where $\|\cdot\|_{I}$ denotes the supremum norm on an interval $I$.

The above definition is parallel to the ones given for nonlinear delay-free impulsive systems in [20] and the ones given for nonlinear impulse-free time-delay systems in [10].

Let $\mathcal{S}$ denote a class of admissible impulsive time sequence. We say that system (1) is input-to-state stable over $\mathcal{S}$ if for any impulsive time sequence in $\mathcal{S}$, system (1) is inputto-state stable; we say that system (1) is uniformly inputto-state stable over $\mathcal{S}$ if for any impulsive time sequence in $\mathcal{S}$, the input-to-state stability property holds with functions $\alpha, \varpi, \gamma_{c}, \gamma_{d}$ that are independent of the choice of the sequence. Inspired by the works in [21] and [22], we denote by $\mathcal{S}_{\min }(\beta)$ the class of impulsive time sequences that satisfy

$$
\inf _{k}\left\{t_{k}-t_{k-1}\right\} \geq \beta
$$

which corresponds to the set of impulse signals with dwell time $\beta$; we denote by $\mathcal{S}_{\max }(\beta)$ the class of impulsive time sequences that satisfy

$$
\sup _{k}\left\{t_{k}-t_{k-1}\right\} \leq \beta,
$$

which corresponds to the set of impulse signals with reversedwell time $\beta$.

\section{INPUT-TO-STATE STABILITY CRITERIA}

In this section, we will establish two theorems which provide sufficient conditions for input-to-state stability of system (1) when one of the delayed impulsive dynamics and the discrete dynamics are input-to-state stable.

Theorem 1: Assume that there exist a function

$$
V: \mathbb{R}^{n} \rightarrow \mathbb{R}^{+}
$$

which is locally Lipschitz, and functions $\alpha_{1}, \alpha_{2}, \chi_{1}, \chi_{2} \in$ $\mathcal{K}_{\infty}$ such that

$\left(\mathbf{H}_{1}\right) \quad \alpha_{1}(|x|) \leq V(x) \leq \alpha_{2}(|x|) ;$ 
$\left(\mathbf{H}_{2}\right)$ There exist scalars $p>1$ and $c>0$ such that

$$
\nabla V(\psi(0)) f\left(\psi, w_{c}\right) \leq-c V(\psi(0))+\chi_{1}\left(\left|w_{c}\right|\right)
$$

for all

$$
\psi \in P C\left([-\tau, 0], \mathbb{R}^{n}\right)
$$

whenever

$$
p V(\psi(0)) \geq V(\psi(\theta))
$$

for $\theta \in[-\tau, 0]$;

$\left(\mathbf{H}_{3}\right)$ There exists $\mu \in[1, p)$ such that

$$
V\left(g\left(x, w_{d}\right)\right) \leq \mu V(x)+\chi_{2}\left(\left|w_{d}\right|\right) .
$$

Then for any given $\beta>0$ satisfying

$$
\mu e^{-c \beta}<1,
$$

system (1) is uniformly input-to-state stable over $\mathcal{S}_{\min }(\beta)$. In particular, when $\mu=1$, system (1) is uniformly input-to-state stable over any impulsive time sequence.

Remark 1: By the results of [9], condition $\left(\mathbf{H}_{2}\right)$ implies that the delayed continuous dynamics are input-tostate stable. But condition $\left(\mathbf{H}_{3}\right)$ implies that the discrete dynamics governing the impulses may be unstable, i.e., the impulses may be destabilizing. So to maintain the input-tostate stability property of the original system, the impulsive interval is required to be large enough to reduce the effect of the impulses. When $\mu=1$, the discrete dynamics are stable for the zero input. In this case, the input-to-state stability property of the original system is not affected by the impulses.

The next theorem provides input-to-state stability conditions for the case when the discrete dynamics are input-tostate stable but the delayed continuous dynamics are not. For this purpose, we need to replace the pair of assumptions $\left(\mathbf{H}_{2}\right)$ and $\left(\mathbf{H}_{3}\right)$ with the following pair of assumptions:

$\left(\mathbf{H}_{4}\right)$ There exist scalars $p>1$ and $c \geq 0$ such that

$$
\nabla V(\psi(0)) f\left(\psi, w_{c}\right) \leq c V(\psi(0))+\chi_{1}\left(\left|w_{c}\right|\right)
$$

for all $\psi \in P C\left([-\tau, 0], \mathbb{R}^{n}\right)$ whenever

$$
p V(\psi(0)) \geq V(\psi(\theta))
$$

for $\theta \in[-\tau, 0]$;

$\left(\mathbf{H}_{5}\right)$ There exists $\mu \in(1 / p, 1)$ such that

$$
V\left(g\left(x, w_{d}\right)\right) \leq \mu V(x)+\chi_{2}\left(\left|w_{d}\right|\right) .
$$

Theorem 2: Assume that there exist a function

$$
V: \mathbb{R}^{n} \rightarrow \mathbb{R}^{+}
$$

which is locally Lipschitz, and functions $\alpha_{1}, \alpha_{2}, \chi_{1}, \chi_{2} \in$ $\mathcal{K}_{\infty}$ such that $\left(\mathbf{H}_{1}\right),\left(\mathbf{H}_{4}\right)$ and $\left(\mathbf{H}_{5}\right)$ hold. Then for any given $\beta>0$ satisfying

$$
\mu e^{c(\beta+\tau)}<1
$$

system (1) is input-to-state stable over $\mathcal{S}_{\max }(\beta)$. Moreover, for the above $\beta$, system (1) is uniformly input-to-state stable over $\mathcal{S}_{\max }(\beta) \bigcap \mathcal{S}_{\min }\left(\beta_{0}\right)$ for any $\beta_{0} \in(0, \beta)$. In particular, when $c=0$, system (1) is input-to-state stable for any impulsive time sequence.

Remark 2: By the results of [7], condition $\left(\mathbf{H}_{3}\right)$ implies that the discrete dynamics governing the impulses are inputto-state stable, i.e., the impulses are stabilizing. But condition $\left(\mathbf{H}_{3}\right)$ also implies that the delayed continuous dynamics may be unstable. So to make the impulsive system be input-tostate stable, the sum of the length of impulsive interval and the time-delay is required to be small enough. When $c=$ 0 , the delayed continuous dynamics are stable for the zero input by Razumikhin Theorem [15]. In this case, the input-tostate stability property of the original system can be achieved without any restriction on the delay and the frequency of the impulses.

\section{Numerical Results and CONClusions}

Consider the state-delayed networked control system of the following form:

$$
\begin{aligned}
\dot{x}(t) & =A x(t)+f\left(x_{t}\right)+B w(t), \\
y(t) & =x(t)+\nu(t), \\
\dot{\hat{x}}(t) & =A \hat{x}(t)+\hat{f}\left(\hat{x}_{t}\right), t \neq t_{k}, \\
\hat{x}_{i}(t) & =\left\{\begin{array}{ll}
y_{i_{k}}\left(t^{-}\right), & i=i_{k}, \\
\hat{x}_{i}\left(t^{-}\right), & i \neq i_{k},
\end{array} \quad t=t_{k}, i=1,2, \ldots, n,\right.
\end{aligned}
$$


where $x(t) \in \mathbb{R}^{n}$ is the system state, $w(t) \in \mathbb{R}^{m}$ is the input disturbance, $y(t) \in \mathbb{R}^{n}$ is the measurement of the state, $\nu(t) \in \mathbb{R}^{n}$ is the measurement noise, $\hat{x}(t) \in \mathbb{R}^{n}$ is the remote estimate of $x(t), f\left(x_{t}\right)$ and $\hat{f}\left(\hat{x}_{t}\right)$ are the unknown nonlinear delayed perturbation of the state and its estimate, respectively, and $\left\{t_{k}\right\}$ is a monotonically increasing transmission time sequence satisfying $t_{k} \rightarrow+\infty$ as $k \rightarrow+\infty$. When $t \in\left(t_{k}, t_{k+1}\right)$, the estimate $\hat{x}(t)$ evolves according to the third equation of (2); and when $t=t_{k}$, a measurement $y_{i k}(t)$ is sent to the remote estimator and this causes the estimate $\hat{x}_{i k}(t)$ to undergo a "jump".

Let

$$
e(t)=\hat{x}_{i}(t)-x_{i}(t)
$$

be the estimation error, which can be described by the following delayed impulsive system

$$
\begin{aligned}
& \dot{e}(t)=A e(t)+\hat{f}\left(\hat{x}_{t}\right)-f\left(x_{t}\right)-B w(t), \quad t \neq t_{k}, \\
& \hat{e}_{i}(t)=\left\{\begin{array}{ll}
\nu_{i_{k}}\left(t^{-}\right), i=i_{k}, \\
e_{i}\left(t^{-}\right), \quad i \neq i_{k},
\end{array} \quad t=t_{k}, i=1,2, \ldots, n .\right.
\end{aligned}
$$

We assume that

$$
\left|f\left(x_{t}\right)-\hat{f}\left(\hat{x}_{t}\right)\right| \leq b \sup _{\theta \in[-\tau, 0]}|x(t+\theta)-\hat{x}(t+\theta)| .
$$

As in [20], we consider a TOD-like protocol [14] to determine the index $i_{k}$ of the measurement $y(t)$ at each transmission time $t=t_{k}$. That is, $i_{k}$ is the index corresponding to the largest

$$
\left|\hat{x}_{i}\left(t_{k}^{-}\right)-y_{i}\left(t_{k}^{-}\right)\right|=\left|e_{i}\left(t_{k}^{-}\right)-\nu_{i}\left(t_{k}^{-}\right)\right|
$$

Define Lyapunov function

$$
V(e)=|e|^{2}
$$

By the results of [20], for every constant

$$
d \in(0, \ln (n /(n-1)))
$$

one can find a function $\chi \in \mathcal{K}_{\infty}$ such that

$$
V\left(e\left(t_{k}\right)\right) \leq e^{-d} V\left(e\left(t_{k}^{-}\right)\right)+\chi\left(\left|\nu\left(t_{k}^{-}\right)\right|\right) .
$$

It follows that system (3) satisfies $\left(\mathbf{H}_{3}\right)$ of Theorem 2 with

$$
\mu=e^{-d}
$$

On the other hand, for some $p>1$, when

$$
p V(e(t)) \geq V(e(t+\theta)), \quad \theta \in[-\tau, 0]
$$

for any $\varepsilon>0$, we have

$$
\begin{aligned}
D^{+} V(e(t)) \leq & e^{T}(t)\left(A+A^{T}\right) e(t) \\
& +2 b|e(t)||e(t-\tau)|-2 e^{T}(t) B w(t) \\
\leq & 2(\mu(A)+b p+\varepsilon) V(e(t)) \\
& +\frac{\left|B^{T} B\right|}{2 \varepsilon}|w(t)|^{2} .
\end{aligned}
$$

There are two possible cases to be considered.

Case 1: $\mu(A)+b<0$. In this case, we can choose $p>1$ and $\varepsilon>0$ such that

$$
\mu(A)+b p+\varepsilon<0 .
$$

It follows from (4) that system (3) satisfies $\left(\mathbf{H}_{2}\right)$ with $c=0$. Therefore, according to Theorem 2, system (2) is input-tostate stable for any transmission time sequence.

Case 2: $\mu(A)+b \geq 0$. In this case, by Theorem 2, the input-to-state stability property of system (2) will depend on the size of the delay $\tau$. We assume that for some given

$$
d \in(0, \ln (n /(n-1))
$$

it holds that

$$
\tau<\frac{d}{2(\mu(A)+b)} .
$$

Choose $\beta>0$ satisfying

$$
-d+2(\mu(A)+b)(\beta+\tau)<0,
$$

i.e.,

$$
\beta+\tau<\frac{d}{2(\mu(A)+b)} .
$$

Then there exist $p>1$ and $\varepsilon>0$ such that

$$
-d+2(\mu(A)+b p+\varepsilon)<0 .
$$

According to Theorem 2, system (2) is input-to-state stable over $\mathcal{S}_{\max }(\beta)$.

To sum up, the primary theoretical findings of this paper are that Razumikhin-type theorems have been established for input-to-state stability of nonlinear impulsive systems with 
time-delay. The numerical results have verified the usefulness of these Razumikhin-type theorems.

\section{ACKNOWLEDGEMENTS}

This work was supported in part by the National Natural Science Foundation of China under Grant 10461001 and the Guangxi Natural Science Foundation (0542032), and in part by a Research Grant from the Australian Research Council and a Research Grant from the University of Western Sydney, Australia.

\section{REFERENCES}

[1] E. D. Sontag, "Smooth stabilization implies coprime factorization," IEEE Transactions on Automatic Control, vol. 34, pp. 435-443, 1989.

[2] E. D. Sontag, "Further facts about input to state stabilization," IEEE Transactions on Automatic Control, vol. 35, pp. 473-476, 1990.

[3] Z. P. Jiang and I. M. Y. Mareels, "A small-gain control method for cascaded nonlinear systems with dynamic uncertainties," IEEE Transactions on Automatic Control, vol. 42, pp. 292-308, 1997.

[4] Z. P. Jiang, A. Teel, and L. Praly, "Small-gain theorem for ISS systems and applications," Mathematics of Control, Signals and Systems, vol. 7, pp. 95-120, 1994.

[5] L. Praly and Z. P. Jiang, "Stabilization by output feedback for systems with ISS inverse dynamics," Systems and Control Letters, vol. 21, pp. 19-33, 1993.

[6] L. Praly and Y. Wang, "Stabilization in spite of matched unmodeled dynamics and an equivalent definition of input-tostate stability," Mathematics of Control, Signals and Systems, vol. 9, pp. 1-33, 1994.

[7] Z. P. Jiang and Y. Wang, "Input-to-state stability for discrete-time nonlinear systems," Automatica, vol. 37, pp. 857-869, 2001.

[8] L. Vu., D. Chatterjee, and D. Liberzon, "Input-to-state stability of switched systems and switching adaptive control," Automatica, vol. 43, pp. 639-646, 2007.

[9] A. R. Teel, "Connections between Razumikhin-type theorems and the ISS nonlinear small gain theorem," IEEE Transactions on Automatic Control, vol. 43, pp. 960-964. 1998.
[10] P. Pepea and Z. P. Jiang, "A Lyapunov-Krasovskii methodology for ISS and iISS of time-delay systems," Systems and Control Letters, vol. 55, pp. 1006-1014, 2006

[11] J. Tsinias, "The concept of 'Exponential input to state stability' for stochastic systems and applications to feedback stabilization, Systems and Control Letters, vol. 36, pp. 221-229, 1999.

[12] D. Nesic and A. R. Teel, "Input-output stability properties of networked control systems," IEEE Transactions on Automatic Control, vol. 49, pp. 1650-1667, 2004.

[13] D. Nesic and A. R. Teel, "Input-to-state stability of networked control systems," Automatica, vol. 40, pp. 2121-2128, 2004.

[14] G. C. Walsh, O. Beldiman, and L. G. Bushnell, "Asymptotic behaviour of nonlinear networked control systems," IEEE Transactions on Automatic Control, vol. 46, 1093-1097, 2001.

[15] K. Gu, V. Kharitonov, and J. Chen, Stability of Time-Delay Systems. Boston, MA: Birkhuser, 2003.

[16] D. D. Bainov and P. S. Simeonov, Systems with Impulse Effect: Stability, Theory and Applications. Chichester: Ellis Horwood, 1989.

[17] T. Yang, Impulsive Systems and Control: Theory and Applications. New York: Nova Science, 2001.

[18] G. Ballinger and X. Liu, "Existence and uniqueness results for impulsive delay differential equations," Dynamics of Continuous, Discrete and Impulsive Systems, vol. 5, pp. 579-591, 1999.

[19] W.-H. Chen, J.-G. Wang, Y.-J. Tang, and X. Lu, "Robust $H_{\infty}$ control of uncertain linear impulsive stochastic systems," International Journal of Robust and Nonlinear Control, in press.

[20] J. P. Hespanha, D. Liberzon, and A. R. Teel, "Lyapunov conditions for input-to-state stability of impulsive systems", Automatica, to appear.

[21] J. P. Hespanha, "Uniform stability of switched linear systems: extensions of LaSalles invariance principle," IEEE Transactions on Automatic Control, vol. 49, pp. 470-482, 2004.

[22] A. S. Morse, "Supervisory control of families of linear set-point controllers Part I. Exact matching," IEEE Transactions on Automatic Control, vol. 41, pp. 1413-1431, 1996. 